\title{
Examination of a scene of crime; do we need to amend section 370 of the Code of Criminal Procedure Act of Sri Lanka?
}

\author{
Niriellage Chandrasiri' \\ The conduct of crin Sri Lanka is most unsatisfactory
}

\section{Introduction}

A scene of crime is a location that has the potential to reveal evidence of the commission of an offence. Examination of a scene of crime can furnish valuable evidence as to the identification of the assailant and the place where the attack occurred, and is the most crucial aspect of the investigation of a suspicious death (1). Sometimes such examination can eliminate suspects. Once the scene of crime is altered it can never be reconstructed.

\section{Team work}

To obtain maximum evidence the investigating team consisting of a crime scene officer (usually a trained policeman), forensic scientist (counterpart in Sri Lanka is a scientific officer from the Government Analyst Department), forensic pathologist (counterpart is the specialist Judical Medical Officer), District Medical Officer, police photographer etc (2), must examine the scene jointly, as early as possible, in an orderly fashion, gather all the data, and record, store and analyse the data before reconstruction of the events of a crime. Sometimes, after examination of the scene of crime by the investigating team, it may be revealed that the incident is a natural one (eg. person collapsing on a beach), thus terminating the search for a criminal and avoiding waste of state funds.

\section{Handling of material at the scene of crime}

The handling of such evidence from its initial location to examination and the presentation of such evidence in a court of law is of paramount importance. This should not be underestimated, undermined or neglected owing to lack of experience or resource constraints, because the rules of evidence in criminal proceedings are very strict (3). This aspect of crime scene investigation is sadly lacking in Sri Lanka, and what is seen at a trial is absolute lack of expertise. Many criminals may have to be discharged due to the inability of the prosecution to fix responsibility on the accused.

\section{Locard's principle .}

The fundamental guiding principle that underlies the approaches to examination of any scene of crime is based on the principle of exchange of material between objects as formulated by Locard. The Locard's principle states that "when two objects come into contact there is always transfer of material from one to the other, and such material may be small or large, may be difficult to detect, nevertheless it occurs and it is the responsibility of the investigating team to gather all such material however small they may be and prove the transference". The perpetrator will take away traces of the victim and the scene, the victim will retain traces of the perpetrator and may leave traces of himself on the perpetrator, and the perpetrator will leave behind traces of himself at the scene.

\section{Legal position in Sri Lanka}

Section 370 of the Criminal Procedure Code Act No 15 of 1979 of Sri Lanka (4) empowers an inquirer into sudden death and a magistrate to examine a scene of a crime. Hence the forensic pathologist, the local medical officer and a forensic scientist are not invited to participate in the initial examination of the scene, thus eliminating experts who have the knowledge and the skill in handling and examining a scene of crime. The inquirers and magistrates are lay people who have had no training in the highly skilled task of examining a scene of crime. They may remove valuable traces of material and may introduce foreign material to the scene due to lack of expertise in examination. Very often we see television clips showing magistrates standing near a body without wearing gloves and an apron, making notes with a police officer, accompained by a large number of inquisitive onlookers with no business at the scene. These well meaning people often destroy the scene. Moving a body can redistribute body fluids (eg. in a rape victim semen can trickle into the anal canal giving the wrong impression of buggery). There have been instances where magistrates have moved bodies (thus making the interpretation of hypostasis difficult) and removed ligatures in cases of strangulation of the neck in the process of examining.

\section{Correct procedure to examine the scene of crime}

How should an investigation team should approach and examine the scene of crime? The moment the local police has informed about a suspicious scene of crime, a responsible officer in that police station should visit the scene and tie a tape ("crime scene tape") around the area to be searched by the investigating team. The idea of cordoning the scene is to prevent contamination and removal of trace material. In addition, an armed police officer should be placed as a guard to prevent any unauthorised persons entering the scene. Often we also see on television politicians accompanied by security guards and several others barging into the scene. The responsible police officer in charge of the investigation must inform the magistrate, the local medical officer, perhaps the judicial medical officer, police photographer and the forensic scientist to visit the scene. All of them should arrive at the scene as early as possible. Some magistrates visit the scene of a crime only 
after leaving the bench, and this is often in the afternoon or late evening. It is the responsibility of the local police to provide sufficient light to conduct the examination even in the night.

\section{Initial assessment of the scene}

Initially the team should obtain information available from the investigating police and discuss strategies to be used in examination of the scene. On arrival at the scene the team should ask the police officer in charge of investigation to ascertain whether the scene has been properly secured and controlled, what was thought to have happened, when it has happened, whether there is a suspect, the persons who have entered the scene of crime and whether the dead person has been identified. Obtaining such information will often make it easier for the team to determine its priorities, and to anticipate the level of contamination and destruction of the scene. The details of background information provided by the police, should not be accepted by the investigators at face value. It should be weighed against the information obtained from the laboratory, and if there is conflicting evidence that should be recorded. The investigating team should be objective and impartial, and report all facts to the court. In large crime scenes involving several deaths, as in a mass disaster, it is necessary to appoint a crime scene coordinator who will be in overall charge. The coordinator should not interfere with the scene of examination, but should be supportive, and act as a buffer between the investigating team and other parties such as magistrate, government ministers, state officers and media personnel.

General survey of the scene is essential initially to develop a systematic approach to the examination of the scene. The team should also take into consideration the weather conditions, time constraints, geography of the place, lighting and the safety precautions. All the personnel involved in the examination should enter and leave the scene from one route. This route should be identified by consultation with all the members of the team. Usually the route is marked by placing duck boards or a polythene sheet over the route. Everybody involved or entering the scene should wear diposable gloves, aprons and overshoes.

\section{Examination of the scene}

There are no hard-and-fast rules applicable to the examination of a scene of crime. A flexible approach should be decided after consultation. Primary considerations should include location of impressions of soles of shoes, fingerprints, tyre marks, contact trace material, abandoned weapons, cigarette butts etc.

\section{Recording}

Recording of a crime scene is an important task and must be accomplished with thoroughness. The recording should be done by obtaining still photography, video recording, making sketches and plans, and making a narrative description.

\section{Still photography}

Still photographs should be taken by a trained police photographer and as early as possible because scenes will not remain undisturbed very long. In fact this should be the first thing to do. The police officers, officers of the court and even the general public may have contaminated the scene. Preliminary discussion will enable the team to advice the photographer as to what photographs should be taken. The initial photographs should be taken from a long distance to include location of the scene, including the body and an immobile landmark, such as a tree or a lamp post. This is to give a general idea of the scene. In a scene where there are several bodies aerial photography may be necessary. The next step is to take medium-distance photographs to record the relative position of the body to the items in the scene. The photographer should ensure that these photographs overlap each other. Finally, the photographer should take close-up pictures of clothes, wounds and other relevant material on the body, and in the scene.

\section{Video recording}

The next step is video recording, and the manner in which it is done is similar to what is done when still photographs are taken. The use of video recording for scenes of crime is gaining importance.

\section{Sketches, plans and maps}

A sketch of the scene is a pictorial representation of the scene and its contents. This is useful in clarifying data obtained from the scene and helps to avoid unnecessary details. A good crime scene sketch compliments still photography and video recording. However, a sketch cannot replace still photography and video recording. If the scene is an enclosed entity such as a room it is necessary to make measurements. The dimensions should include those of the room, doors and windows, distances from relevant objects, distances between objects and the distance of the body to a fixed point. All the distances should be measured in two axes at right angle to each other. The sketch should indicate north-south position obtained by using a compass.

\section{Searching techniques}

The searching techniques available are, line searching (possible if there are large number of police officers), zonal searching (useful when portions are fragmented as in an explosion), grid searching, spiral searching (is useful when the terrain is difficult), and strip searching (useful when there is a limited number of police officers). Whatever method is employed the searchers must be fully briefed by the person in charge of crime scene investigation. The search must be stopped if something interesting is located and it must be immediately photographed and collected.

\section{Collection of evidence}

This is a very important phase in the examination of a scene of crime. When in doubt collect any potential item as its relevance may become apparent later. It is better to 
collect too much than too little. Experienced crime scene officers often achieve a correct balance, thus minimising the time spent in collecting evidence. Any item which could be lost or affected by wind, rain, people etc, items at the entry and exit points and path, items under the body, any item which may give a clue to the identity of the deceased should be collected initially. Contact traces should be taken before dusting for fingerprints. Polished floors need to be examined with oblique lighting to locate latent shoe impressions. Blood stains should also be collected before taking fingerprints. All the items should be labelled at the time of collection either by description, numerical sequence, or both, to maintain continuity. In the case of large or complex crime scenes, consideration should be given to a master $\log$ of exhibits or the employment of an exhibits officer.

\section{Labelling and packing}

All items should be labelled at the time of collection by description and numerical sequence. Correct labelling allows identification of the exhibits right through their presentation in court. Labels should be brief, indicate the nature of the item, time and date, and the name of the person collecting, and they should be securely fixed to the container or the package that contains the item. Use of thick brown paper bags is preferred to polythene, which enhances fungal growth.

\section{Safety measures}

The investigating team should not commence searching scenes of fire, where death has been caused by bomb explosions and eletrocution etc, before these locations have been examined by the appropriate experts and declared safe. Special care should be taken not to get contaminated with blood and other biological fluids to prevent contamination with blood related viruses. Use of safety glasses, goggles, face masks and protective aprons will prevent such contamination.

\section{Conclusions}

Examination of a scene of crime is a highly skilled task and should be conducted by a team of experts who have the knowledge and skill to collect necessary data from the scene. It is time that section $\mathbf{3 7 0}$ of the Criminal Procedure Code Act be amended either to delete the necessity of the inquirer or magistrate to examine a scene of crime, or to include the medical officer in this section. Taking photographs of the entire body from front and back will indicate accurately the number and position of all wounds, and the need for an inquirer or the magistrate to examine the scene is redundant. One needs medical training to differentiate between peri-mortem (wounds caused at the time of death) and post-mortem wounds and magistrates and inquirers have often mistaken postmortem wounds as peri-mortem wounds, causing confusion in interpretation. It is obvious to those who have been involved in crime scene examinations in Sri Lanka that the manner in which the investigating police conduct crime scene examinations is highly unsatisfactory. There should be trained police officers to examine a scene of crime. Police stations should be provided with cameras and material necessary for packaging of exhibits and the medical officer or the forensic pathologist must necessarily be involved with the investigating police at the initial examination of the scene of crime.

\section{References}

1. Vanezis P, Busutill A. Suspicious death scene investigation. London: Amold, 1996.

2. Simpson K. Police: the investigation of violence. London: Macdonald and Evans 1978.

3. Clement GJ, Ranson LD. Cranio-facial identification in forensic medicine. London: Amold, 1998.

4. Code of Criminal Procedure. Act No. 15 of 1979, p 146.

\title{
Antiseptics in wound management
}

\begin{abstract}
All antiseptics are cytotoxic! They damage cellular elements and the microcirculation of the wound. Although antiseptics may have a role in the topical management of heavily contaminated acute traumatic ulcers, they are often inappropriately used for long periods of time on chronic ulcers. The need to sterilise a chronic ulcer to achieve healing is unproven and there is thus little evidence to support the ongoing use of antiseptics in chronic wound management.
\end{abstract}

MacLellan DG. Chronic wound management. Australian Prescriber 2000; 23 : 6-9. 